\title{
Incipient Berezinskii-Kosterlitz-Thouless transition in two-dimensional coplanar Josephson junctions
}

\author{
D. Massarotti, ${ }^{1,2, *}$ B. Jouault, ${ }^{3}$ V. Rouco, ${ }^{4}$ S. Charpentier, ${ }^{5}$ T. Bauch, ${ }^{5}$ A. Michon, ${ }^{6}$ A. De Candia, ${ }^{2,4}$ P. Lucignano, ${ }^{2,4}$ \\ F. Lombardi, ${ }^{5}$ F. Tafuri, ${ }^{1,2}$ and A. Tagliacozzo ${ }^{2,4,7}$ \\ ${ }^{1}$ Dipartimento di Ingegneria Industriale e dell'Informazione, Seconda Università di Napoli, I-81031 Aversa (CE), Italy \\ ${ }^{2}$ CNR-SPIN UOS Napoli, Monte S. Angelo-Via Cintia, I-80126, Napoli, Italy \\ ${ }^{3}$ Laboratoire Charles Coulomb (L2C), UMR 5221 CNRS-Université de Montpellier, F-34095, Montpellier, France \\ ${ }^{4}$ Dipartimento di Fisica "E. Pancini”, Università di Napoli Federico II, Monte S. Angelo-Via Cintia, I-80126 Napoli, Italy \\ ${ }^{5}$ Department of Microtechnology and Nanoscience, Chalmers University of Technology, SE-412 96 Göteborg, Sweden \\ ${ }^{6}$ Centre de Recherche sur l'Hétéro-Epitaxie et ses Applications (CRHEA), CNRS, rue Bernard Grégory, O6560 Valbonne, France \\ ${ }^{7}$ INFN, Laboratori Nazionali di Frascati, Via E. Fermi, Frascati, Italy
}

(Received 11 May 2016; revised manuscript received 20 July 2016; published 31 August 2016)

\begin{abstract}
Superconducting hybrid junctions are revealing a variety of effects. Some of them are due to the special layout of these devices, which often use a coplanar configuration with relatively large barrier channels and the possibility of hosting Pearl vortices. A Josephson junction with a quasi-ideal two-dimensional barrier has been realized by growing graphene on $\mathrm{SiC}$ with $\mathrm{Al}$ electrodes. Chemical vapor deposition offers centimeter size monolayer areas where it is possible to realize a comparative analysis of different devices with nominally the same barrier. In samples with a graphene gap below $400 \mathrm{~nm}$, we have found evidence of Josephson coherence in the presence of an incipient Berezinskii-Kosterlitz-Thouless transition. When the magnetic field is cycled, a remarkable hysteretic collapse and revival of the Josephson supercurrent occurs. Similar hysteresis are found in granular systems and are usually justified within the Bean critical state model (CSM). We show that the CSM, with appropriate account for the low-dimensional geometry, can partly explain the odd features measured in these junctions.
\end{abstract}

DOI: 10.1103/PhysRevB.94.054525

\section{INTRODUCTION}

Superconducting hybrid junctions can now be obtained combining superconductors with functional barriers as semiconductors [1], nanowires with spin orbit coupling [2], graphene [3,4], topological insulators [5,6], and ferromagnets $[7,8]$ made superconducting by the proximity effect. These devices often use a coplanar layout with almost twodimensional (2D) flakes or nanowires as barriers [1,9-13]. The geometry of the device and the nature of the interfaces may favor the appearance of exotic effects ranging from Majorana fermions [14,15] to topological superconductivity [6] and anomalous Josephson effect [16]. In this paper we will show how the emerging class of 2D extended barriers can promote Josephson coherence in the presence of an incipient Berezinskii-Kosterlitz-Thouless [17,18] transition, which is indeed supposed to be a peculiarity of $2 \mathrm{D}$ superconducting systems. In this case a 2D barrier is made superconducting by proximity effect. To this aim we have realized graphene Josephson junctions (GJJs), obtained using a graphene barrier deposited on $\mathrm{SiC}$ by chemical vapor deposition (CVD) rather than the standard technique of an exfoliated flake [19]. Graphene on $\mathrm{SiC}$ is highly homogeneous at the centimeter scale. Thus relatively simple lithography processes allow us to obtain thousands of devices on the same wafer [20]. This specific type of graphene growth guarantees having devices with the desired geometry, i.e., a 2D barrier of suitable lateral dimensions to host 2D vortex structures. Large graphene samples deposited as a thin film offer enough room to host extremely spread out $2 \mathrm{D}$ vortex entities. Vortex pinning is

\footnotetext{
*Corresponding author: dmassarotti@na.infn.it
}

expected to occur because of interface impurities between the graphene sheet and the thin superconducting electrode.

In this paper we show how the Josephson coherence is observed along with very unusual features like the persistence of a small residual resistance and, even more surprising, the appearance of a hysteresis in magnetic field, manifesting in collapses and revivals of the supercurrent depending on the direction of the magnetic sweeping (see Fig. 4). This points to Josephson conduction in the presence of an incipient Berezinskii-Kosterlitz-Thouless (BKT) transition at sub-Kelvin temperature.

Our results are a fundamental step towards scalability for GJJs, and for our goals it offers the additional advantage of comparing junctions fabricated on exactly the same barrier. This can be extremely useful to have further insights on the electrodynamic response and the nature of dissipation of GJJs, which need to be considered in the actual rush of more performing devices in the ballistic limit, to exploit the unique properties of Andreev reflection in graphene [21-27].

In Sec. II we report on the preparation of the sample. A consistent interpretation of the phenomenology described in Secs. III-V requires frames which go beyond the usual Josephson S/N/S paradigm. The superconducting proximity is quite unique in our samples. The Josephson supercurrent is always accompanied by a small resistance, but it drops to a very low value very fast, as soon as a very weak orthogonal magnetic field $H$ is applied, apparently without loosing Josephson phase coherence. Meanwhile, the resistance increases from a few Ohms to values of the order of $400 \Omega$. The situation does not change if the field is further increased, but, as soon as we invert the sweeping direction at any magnetic field value below a threshold field, the Josephson supercurrent has an unexpected revival, in the presence of a remanent 
magnetization. Simultaneously, the resistance drops again. All these observations are consistent with a special regime of the proximity effect, expected to occur only in two dimensions and in the presence of pinning of vortices.

Similar hysteresis in the magnetization or in the critical current of granular systems is found in type II superconductors and is usually justified within the Bean critical state model (CSM) [28]. The CSM can provide a qualitative interpretation of the magnetization process, which takes place in the $\mathrm{Al} / \mathrm{Ti}$ islands covering the graphene, and of the measured hysteresis. Support for this interpretation comes from the fact that metal decorated graphene sheets have been fabricated [29] and their superconducting phase transition has been classified as of the BKT type $[17,18]$. Cooling down the devices below the critical temperature of the electrodes $\left(T<T_{c}^{A l} \simeq 1.1 \mathrm{~K}\right)$ in zero field apparently leads to incipient superconductivity in the graphene layer.

The plots of the resistance vs temperature presented in Sec. III support a qualitative interpretation of the measured phenomena within the BKT theory (see Fig. 3) at low temperatures. In this regime vortex-antivortex $(v-\bar{v})$ pair unbinding and flux-flow resistance across the junction are dominant phenomena. We have measured our samples down to $T=280 \mathrm{mK}$ which is a much higher temperature than the expected BKT critical temperature, $T_{\mathrm{BKT}}$. Features of the BKT incipient transition survive in the crossover to a paraconductivity regime at $T_{\mathrm{BKT}}<T<T_{c}^{A l}$. The Josephson supercurrent modulated by the magnetic field is accompanied by the flow of vortices, which induces a finite slope in the supercurrent branch. An analysis of the flux flow resistance points to large Pearl vortices, pinned to the impurities in the CSM phase. Magnetic screening is very weak and correlations at intermediate distances induced by the long range repulsive interaction can be strong, particularly when the applied orthogonal magnetic field is very small. All of this is discussed in Secs. IV and V.

In Sec. VI the Fraunhofer pattern of the Josephson conduction is discussed. By ignoring details of the microscopic vortex structure and dynamics, a macroscopic approach to the diamagnetic screening currents, based on the solution of the London equation in a quasi-2D contact, can reasonably account for the measured pattern if flux focusing effects are assumed in the planar thin-film weak link [30]. Sections VII and VIII contain a detailed summary and the conclusions, respectively.

\section{SAMPLE PREPARATION}

The graphene layer was grown by CVD on the silicon face of a $\mathrm{SiC}$ substrate. The semi-insulating $\mathrm{SiC}$ substrate has an extremely small, negligible conductivity. The quality of the monolayer structure was carefully investigated by Raman and angle resolved photoemission spectroscopy [19] (see Refs. [31,32] for details). Before lithography, the mobility at room temperature is $\mu \simeq 500 \mathrm{~cm}^{2} / \mathrm{V}$ s. The graphene is $p$ doped, $p \simeq 5 \times 10^{12} \mathrm{~cm}^{-2}$. This $p$ doping is induced by the hydrogenation of the interface between graphene and $\mathrm{SiC}$ [32].

The planar Josephson devices are fabricated by conventional $e$-beam lithography. The graphene junctions are patterned by oxygen plasma. Then, the contacts are deposited

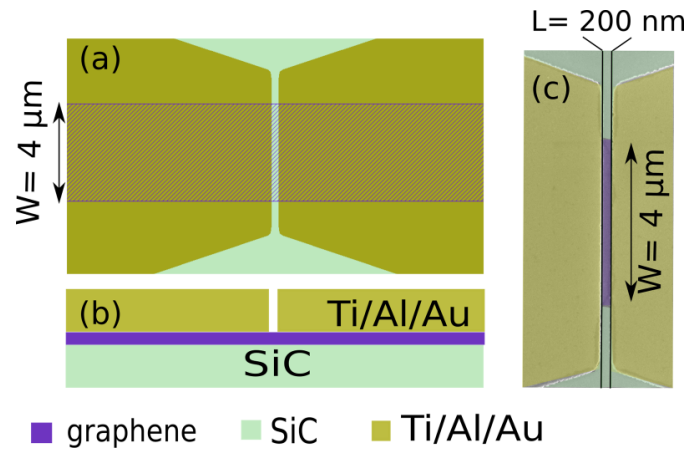

FIG. 1. Josephson devices with large 2D contact barriers. (a) Large sketch of the planar devices. (b) Sketch of the graphene junction. (c) Scanning-electron micrograph of a $L \approx 200 \mathrm{~nm}$ short junction with artificial colors.

(see Fig. 1). They consist of a $5 \mathrm{~nm}$ interfacial layer of titanium, which ensures good electrical contact to graphene, an 80-nm-thick aluminium layer, and a 3-nm-thick gold layer. It has been reported that $\mathrm{Al}$ on top of graphene could induce a strong $n$ doping [33]. As the pristine graphene is initially $p$ doped, these $\mathrm{Al} / \mathrm{graphene}$ junctions could be suitable for fabricating $n-p-n$ junctions, with appropriate protocols which include gates. In our devices, the gap $L$ between the electrodes remains relatively large, ranging from $200 \mathrm{~nm}$ to $600 \mathrm{~nm}$. The width of the junctions is fixed at $W=4 \mu \mathrm{m}$. Charge transport is most likely diffusive. Thus Fabry-Perot resonances and Klein tunneling are not expected to be present.

These junctions have a major difference with graphene junctions obtained by the exfoliation technique. While the geometrical junction area is $\mathcal{A}_{j}=W \times L$, here the remaining graphene area below the superconducting $\mathrm{Al}$ contacts is massive. On purpose, we left a graphene area $\simeq 10^{3} \mu \mathrm{m}^{2}$ under each of the two Al contacts. A part of these areas is visible in Fig. 1(a). For the measurements, the samples are thermally anchored to the cold stage of a ${ }^{3} \mathrm{He}$ cryostat equipped with EMI filters at room temperature, $\mathrm{RC}$ filters at the $1-\mathrm{K}$ pot stage and copper powder filters at the sample stage [34,35]. Current-voltage (I-V) characteristics of different junctions have been measured as a function of temperature and magnetic field. Measurements of resistance as a function of temperature, $R(T)$, and magnetic field, $R(H)$, have been performed with standard low frequency lock-in techniques using low excitation currents, in the range 5-10 nA.

Table I collects measured and fitted parameters of some of the studied junctions. For the shortest junction (J200-4, $L=$ $200 \mathrm{~nm}$ ), the I-V curves are reported at various temperatures in Fig. 2. We measure a finite slope in the I-V characteristics close to zero voltage, even at the lowest temperatures. The linear part is followed by a bending which is characteristic of the resistively shunted junction (RSJ) model. The critical current $I_{c}$ as a function of an externally applied magnetic field is estimated through the RSJ model (see inset of Fig. 2), suitably used for an overdamped Josephson junction [36] in series with a resistance. We attribute the finite slope in the supercurrent branch to the presence of fluctuating broken vortex-antivortex pairs which cannot be described appropriately within the RSJ framework or more refined arguments based on phase diffusion $[34,35,37,38]$ (see Secs. IV and V). Indeed, our RSJ 
TABLE I. Main parameters for the investigated devices: name, length $L$, normal resistance $R_{N}$, critical current $I_{c}$ at $T=280 \mathrm{mK}$, mean field resistance $R_{0}$, mean field critical temperature $T_{c 0}$, BKT temperature, dimensionless parameter $b$ of Eq. (2).

\begin{tabular}{lccccccc}
\hline \hline Name & $\begin{array}{c}L \\
\mathrm{~nm}\end{array}$ & $\begin{array}{c}R_{N} \\
\Omega\end{array}$ & $\begin{array}{c}I_{c} \\
\mathrm{nA}\end{array}$ & $\begin{array}{c}R_{0} \\
\mathrm{k} \Omega\end{array}$ & $\begin{array}{c}T_{c 0} \\
\mathrm{~K}\end{array}$ & $\begin{array}{c}T_{\mathrm{BKT}} \\
\mathrm{mK}\end{array}$ & $b$ \\
\hline $\mathrm{J} 200-1$ & 200 & 720 & 4 & 8.5 & 0.23 & & \\
$\mathrm{~J} 200-2$ & 200 & 425 & 5 & & & & \\
$\mathrm{~J} 200-3$ & 200 & 410 & 10 & 1.4 & 0.35 & 130 & 6.1 \\
$\mathrm{~J} 200-4$ & 200 & 470 & 50 & 1.0 & 0.5 & 135 & 8.6 \\
$\mathrm{~J} 300-3$ & 300 & 370 & 30 & 1.3 & 0.38 & 175 & 7.2 \\
$\mathrm{~J} 400-1$ & 400 & 650 & 0 & 16.0 & 0.285 & & \\
$\mathrm{~J} 600-1$ & 600 & 440 & 0 & & & & \\
\hline \hline
\end{tabular}

fit points to an effective temperature which is larger than the base temperature.

\section{TEMPERATURE DEPENDENCE OF THE RESISTANCE AT ZERO $H$ FIELD}

In Fig. 3(b) the $R(T)$ curves in zero field cooling (ZFC) are plotted for the three junctions, which appear to be on the verge of a BKT transition at low temperatures (J200-3, J200-4 and J300-3). There is first a partial drop in the resistance which occurs just above $1 \mathrm{~K}$ that can be attributed to the transition to the superconducting state of the $\mathrm{Al} / \mathrm{Ti}$ contacts. Below $1 \mathrm{~K}$ we identify two different regimes which we attribute to Aslamazov-Larkin (AL) paraconductivity and incipient BKT transition. The two regimes are described here below.

\section{A. AL paraconductivity regime}

The high temperature partial drop in the resistance occurring as a broad transition between $1 \mathrm{~K}$ and $0.5 \mathrm{~K}$ is consistent with a manifestation of paraconductivity in the graphene layer. Thermal fluctuations in Cooper pair formation enhanced by proximity allow us to define a mean field pairing temperature $T_{c 0}$, below which the amplitude of the superconducting condensation of pairs is expected to be finite. The drop at zero magnetic field $\left(T>T_{c 0}\right)$ can be fitted

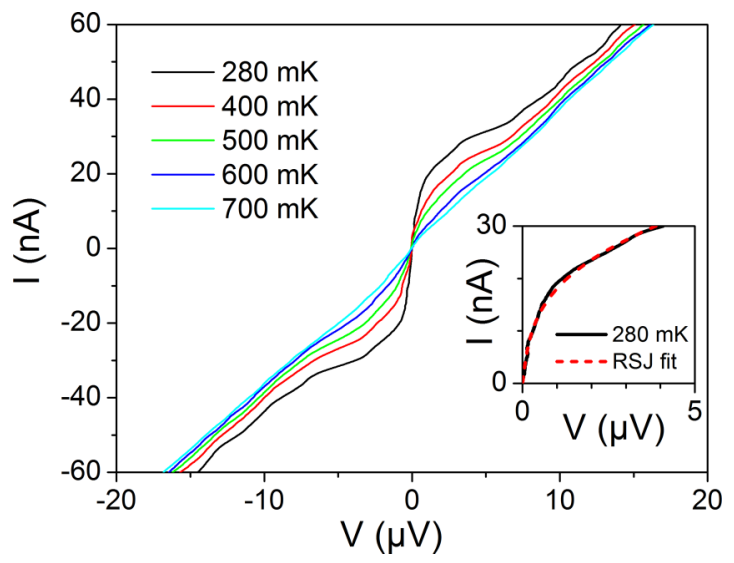

FIG. 2. I-V characteristics measured at various temperatures for the J200-4 junction. The inset shows the RSJ fit (red dashed line) of the I-V curve measured at $280 \mathrm{mK}$ (black full line).
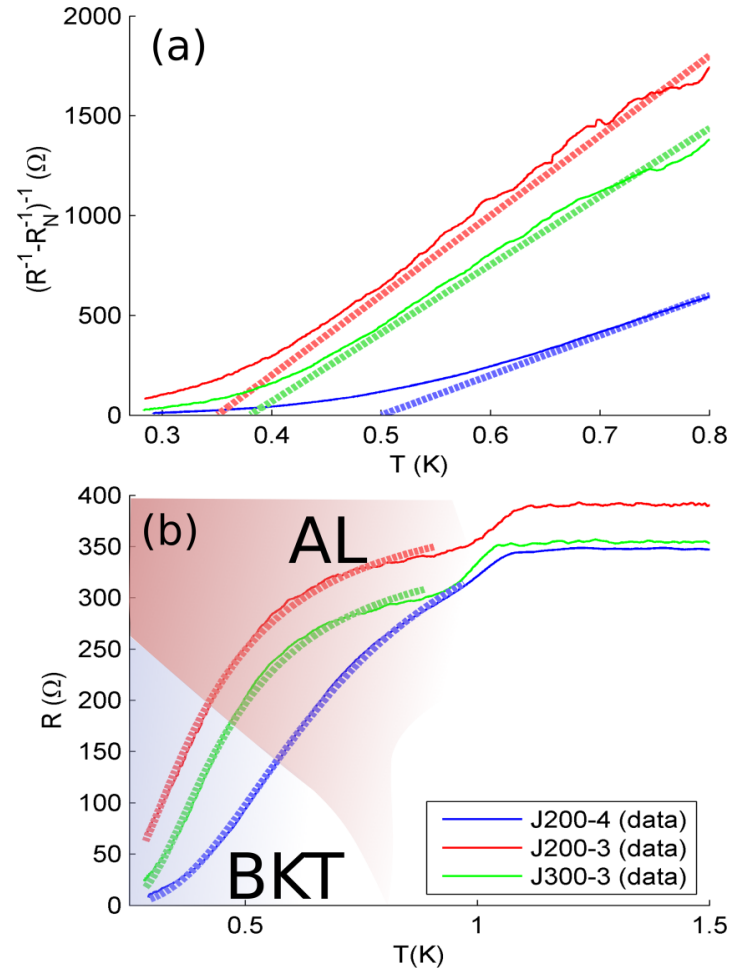

FIG. 3. $R(T)$ curves of three junctions (J200-3, J200-4 and J3003 ). (a) The resistances are rescaled as $\left(R(T)^{-1}-R_{N}^{-1}\right)^{-1}$ (thin solid curves). According to the AL theory, see Eq. (1), interception of the linear fits (thick solid lines) with the $x$ axis gives the mean field pairing temperature $T_{c 0}$. (b) Fit of the resistances $R(T)$ of the three junctions given above, using Eq. (2), with parameters from Table I (thick broken lines). The domain of validity of the AL model is shaded in red. At lower temperature, the assumed domain of validity of the BKT theory is shaded in light blue.

by the conductivity change $\delta \sigma(T) \approx \ln ^{-1}\left(T / T_{c 0}\right)$ typical of Aslamazov-Larkin (AL) fluctuation-enhanced conductivity in two dimensions [39]. We replot the data as $\left(R(T)^{-1}-R_{N}^{-1}\right)^{-1}$ [thin solid lines in Fig. 3(a)]. According to the AL theory, linear fits [thick solid lines in Fig. 3(a)] give the value for the hypothetical mean field pairing temperature $T_{c 0}$ at the interception with the $x$ axis [40]:

$$
\left(R(T)^{-1}-R_{N}^{-1}\right)^{-1}=R_{0}\left(T-T_{c 0}\right) / T_{c 0} .
$$

Here $R_{0}$ is a fitting parameter predicted to be of the order of $16 \hbar / e^{2}$ while $R_{N}$ is the normal resistance given by the slope of the I-V characteristics at large voltages. The parameters $R_{0}$ and $T_{c 0}$ of the various measured junctions are reported in Table I. The mean-field pairing temperatures $T_{c 0}$ which we obtain in most of the measured samples fall within a small range of temperature $T=0.23-0.5 \mathrm{~K}$.

\section{B. BKT incipient transition}

In superconducting films, below the mean field temperature $T_{c 0}$, the pairing amplitude is finite but overall superconducting phase coherence cannot be established due to thermal fluctuations. By further lowering the temperature, we enter a crossover region towards a BKT transition typical of dirty 
thin films. The low temperature behavior of $R(T)$ deviates from the paraconductivity regime which is power-law like and enters an exponential-law behavior as shown in Fig. 3(b). At $T_{c 0}>T>T_{\mathrm{BKT}}$, the global phase coherence is destroyed by thermally induced phase fluctuations in the form of free vortices which produce dissipative conduction due to a finite flux flow resistance $[17,18]$. In our case, the wide graphene sheet, as well as the large overlap area between the graphene and the $\mathrm{Al} / \mathrm{Ti}$ pads, provides enough space for hosting even extremely extended vortices (such as Pearl vortices appearing in very thin films). The fit of the measured $R(T)$ by using the interpolation formula quoted by Halperin and Nelson [41], which is valid for $T>T_{\mathrm{BKT}}$ :

$$
[R(T)]^{-1}=\frac{0.37}{b}\left[R_{N}\right]^{-1} \sinh ^{2}\left[\left(\frac{b t_{c}}{t}\right)^{1 / 2}\right],
$$

is quite successful over more than one decade of resistance values [thick broken lines in Fig. 3(b)] and covers the full temperature crossover including the $\mathrm{AL}$ regime. Given $T_{c 0}$ as extracted from Fig. 3(a), the two fitting parameters here used are $T_{\mathrm{BKT}}$ and $b$. In Eq. (2) $t_{c}=\left(T_{c 0}-T_{\mathrm{BKT}}\right) / T_{\mathrm{BKT}}$ and $t=\left(T-T_{\mathrm{BKT}}\right) / T_{\mathrm{BKT}}$ appear. The 0.37 prefactor is chosen by Halperin and Nelson to match with the AL linear dependence of Eq. (1) with $R_{0}=16 \hbar / e^{2}$. Although our fitted values of $R_{0}$ are at least one order of magnitude smaller than this value derived from the Ginzburg-Landau theory, we have kept it unaltered as the fit appears to be rather insensitive to it. The dimensionless parameter $b$ is related to the ratio between the loss in condensation energy at a vortex core and the superfluid stiffness. It is remarkable that the values of $b$ reported in Table I are of the same order as the accepted values for the 2D-XY model [40] in indium oxide films. The fitted temperatures $T_{\mathrm{BKT}}$, of the order of $0.1 \mathrm{~K}$, as well as the $b$ and $R_{0}$ values are reported in Table I.

\section{HYSTERETIC MAGNETIC FIELD DEPENDENCE OF THE JOSEPHSON CURRENT}

Cooling the junctions with $L<400 \mathrm{~nm}$ down to $T=280$ $\mathrm{mK}$ in ZFC, a Josephson current is established, with $I_{c} \approx$ $50 \mathrm{nA}$, notwithstanding the fact that there are thermal fluctuations which produce free vortices and antivortices in the graphene sheet $\left(T>T_{\mathrm{BKT}}\right)$. In Fig. 4 we report various hysteresis loops of $I_{c}$ with an externally applied magnetic field $H_{a}$ for the junction $\mathrm{J} 200-4$ of smaller area $\mathcal{A}_{J 200} \simeq 1 \mu \mathrm{m}^{2}$. For this junction, $L=200 \mathrm{~nm}$.

The sweeping of the applied field $H_{a}$ is: $H_{a}=-H_{m} \rightarrow H_{m}$ (red curve) and $H_{a}=H_{m} \rightarrow-H_{m}$ (blue curve). Here $H_{m}$ is defined as the maximum attained $\left|H_{a}\right|$ field before initiating the decreasing in the sweep. We find that $I_{c}$ rapidly drops to very low values with increasing field $\left|H_{a}\right|$ in both positive and negative directions of the sweep. By contrast, in decreasing $\left|H_{a}\right|, I_{c}$ appears to recover and is strongly sensitive to magnetic field variation displaying fluctuations between various runs [see, e.g., Fig. 4(a), close to $H_{a} \simeq 10 \mathrm{Oe}$. This is a robust feature, as demonstrated by the various measurements of the same junction presented in Figs. 4(a)-4(e) in which $H_{m}$ is varied.

The junctions reported in the present work are characterized by nonhysteretic IV curves with a finite slope in the super-
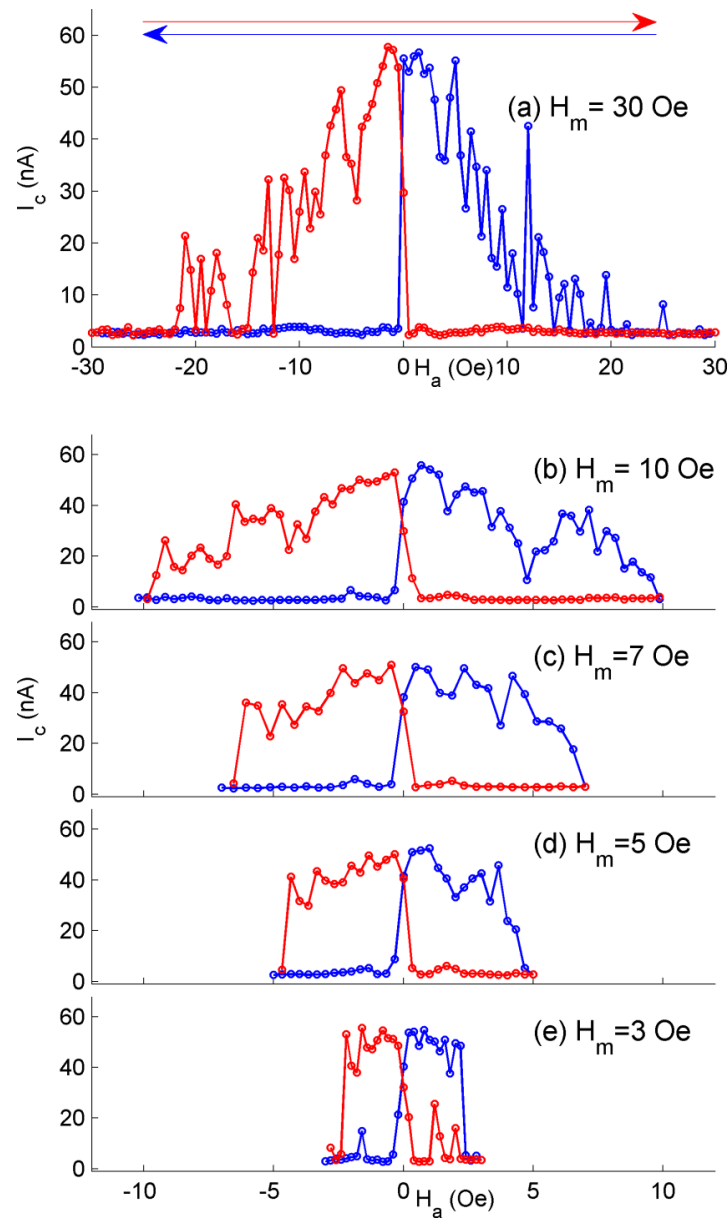

FIG. 4. Critical Josephson current $I_{c}$ as a function of the applied magnetic field $H_{a}$ for the J200-4 junction. In all panels, the red (blue) color refers to sweeps performed from negative to positive (positive to negative) magnetic fields. Magnetic sweeps range up to $H_{m}=30 \mathrm{Oe}$ (a), $10 \mathrm{Oe} \mathrm{(b),} 7 \mathrm{Oe}$ (c), $5 \mathrm{Oe}(\mathrm{d}), 3 \mathrm{Oe}(\mathrm{e})$. The arrows on top indicate the direction of the magnetic field variation.

conducting branch, as shown in Fig. 2. In the framework of the RSJ model, this phenomenology could be explained by considering diffusion of the phase particle along the washboard potential, when the Josephson energy is of the same order of the thermal energy [36]. Indeed, phase diffusion has been observed recently in graphene-based JJs [42]. Nevertheless, the measurements in the presence of applied magnetic field clearly indicate that phase diffusion is not the main dissipation process. Indeed, the magnetic field changes the amplitude of the supercurrent (estimated by RSJ fit) and modulates the finite slope of the supercurrent branch in a very anomalous way, since it depends on the magnetic field sweep direction. Modulation of the critical current and of the finite slope of the supercurrent branch are shown in Fig. 4 and in panel (a) and in panel (b) of Fig. 5, which reports the dependence of the resistance at zero bias as a function of magnetic field. These last experimental observations cannot be explained within the RSJ model, and within any kind of phase diffusion process [34,35,37,38]. Their interpretation will be addressed in Sec. V.

In a magnetic field parallel to the graphene flake we have measured the same hysteresis on a magnetic field scale 

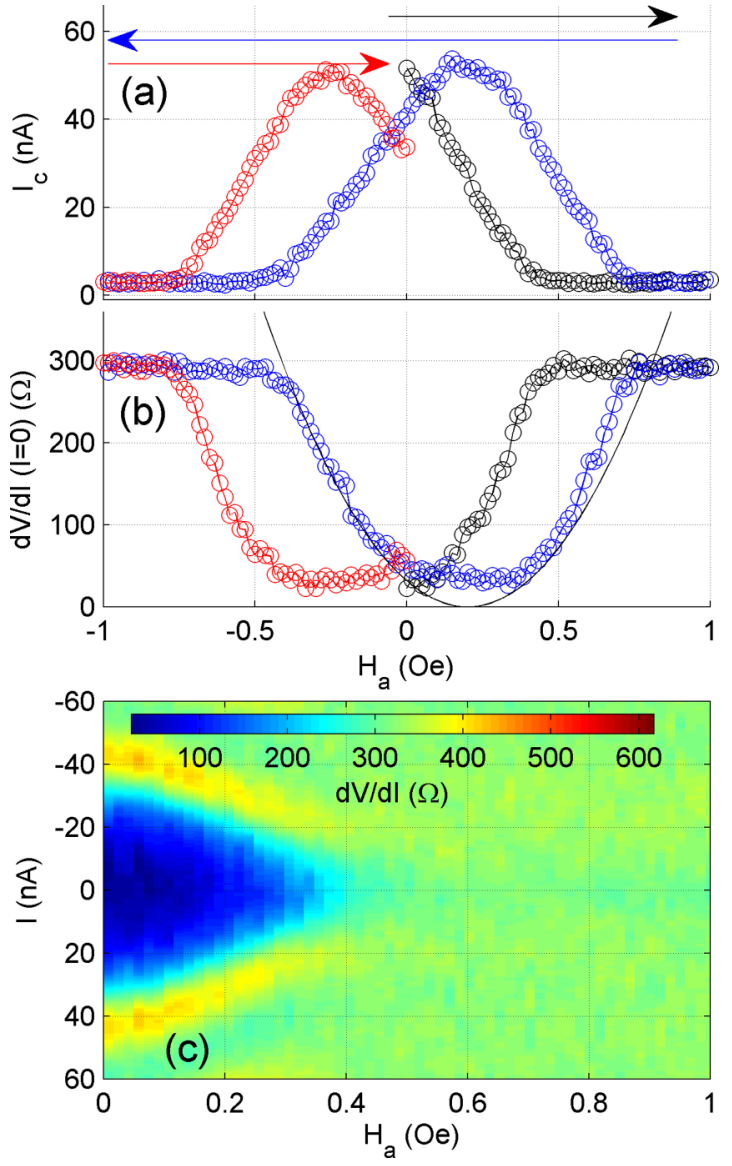

FIG. 5. Zoom on the hysteresis of Fig. 4 at low magnetic fields $\left(H_{m} \leqslant 1 \mathrm{Oe}\right)$ for the J200-4 junction, taken at $T=280 \mathrm{mK}$. (a) Estimated critical current during a magnetic sweep (sweeping direction given by the arrows). Black circles: the sweep starts at $H_{a}=0 \mathrm{Oe}$ at ZFC and goes up to $H_{a}=1 \mathrm{Oe}$. No flux is trapped initially in this first sweep after the cool down. Blue circles: the magnetic field is swept from 1 Oe to -1 Oe. Red circles: the magnetic field is swept back from -1 Oe to 0 Oe. (b) The differential resistance at $I=0$ recorded when the magnetic field is swept from 0 to $1 \mathrm{Oe}$ (open dark circles), from 1 Oe to -1 Oe (open blue circles), and from -1 Oe to $0 \mathrm{Oe}$ (open red circles). The black curve is a parabola, which serves as a guide for the eye. (c) Color map of the differential resistance $d V / d I(H, I)$ recorded during the first sweep after ZFC, from 0 Oe to 1 Oe. It corresponds to the black circles of panel (a), i.e., it reports the first collapse of the critical current $I_{c}$. For $H_{a}>$ $0.4 \mathrm{Oe}$, viscosity $\eta \propto H$ [see Eq. (4)] and the critical state model applies, with $R \simeq 300 \Omega$.

enlarged by a factor $\simeq 100$. As some undesired tilting of the sample cannot be excluded, we conclude that the hysteresis is generated by a small spurious orthogonal $H$ component due to a misalignment of the coil in the parallel geometry. This confirms that the electronic properties of the system are not appreciably affected by a field $H_{a}$ parallel to the flake, while the orthogonal component of the field is the main actor. We can also exclude magnetization effects of nonsuperconducting origin in the $\mathrm{Al}$ contacts. Indeed, in the normal phase $\left(T>T_{c}^{A l}\right)$ we do not measure any hysteresis. The hysteresis in the supercurrent is strongly dependent on the geometry of the weak link, as, by excluding the weak link and contacting one of the $\mathrm{Al}$ islands alone, no hysteresis appears in the supercurrent.

\section{COLLAPSE OF THE JOSEPHSON SUPERCURRENT CLOSE TO ZERO MAGNETIC FIELD}

In this section, we propose an interpretation of the unusual collapse of the Josephson critical current $I_{c}$, close to zero field, as well as of the revival at the inversion point. $I_{c}\left(H_{a}\right)$ and the corresponding $R\left(H_{a}\right)$ are reported in Figs. 5(a) and 5(b) at a magnetic field scale much smaller than the one appearing in Fig. 4. In Fig. 5(a), the first drop of $I_{c}$ with the increasing of $H_{a}$ after ZFC is marked by open black dots. From this curve we extract the magnetic field value $H_{f}=0.45$ Oe at which the $I_{c}$ collapse is completed. Furthermore, the maxima of $I_{c}$ (and the minima of $R$ ) in the back sweeps are shifted to $\left|H_{a}\right| \simeq 0.25 \mathrm{Oe}$, where the sign depends on the sweep direction. Additionally, comparison of Fig. 5(a) with Fig. 5(b) reveals that in all the magnetic field sweeps there is a direct relation between $I_{c}$ and the residual magnetoresistance $R(H)$.

The hysteresis observed in Figs. 5(a) and 5(b) shares two important features with the hysteresis which is commonly observed in the magnetoresistance of granular superconductors:

(i) for the same value of $H_{a}$, the resistance in the decreasing $\left|H_{a}\right|$ curve is lower than that in the increasing $\left|H_{a}\right|$ curve;

(ii) the minimum of $R$ is obtained before $\left|H_{a}\right|$ reaches zero in decreasing $\left|H_{a}\right|$. The usual model to explain these features in granular superconductors is the so-called two-level critical state model, developed in Ref. [43], in which superconducting grains trap and pin vortices, inducing an hysteresis, whereas the vortex dynamic at the grain boundaries gives rise to a finite resistance. In our view, pinning centers are present under the $\mathrm{Al}$ pads and the observed incipient BKT transition signals the presence of vortices in the same area. In the simplest interpretation, the areas under the $\mathrm{Al}$ pads correspond to two separated grains, whereas the bare graphene junction corresponds to the boundary between these two grains. We cannot exclude a more complex picture, in which the areas under the Al pads are themselves constituted of several smaller grains.

Coming back to Fig. 5, we try now to understand why the collapse of $I_{c}$ with increasing $\left|H_{a}\right|$ is so drastic. A viscosity of the vortex liquid can be extracted from the magnetoresistance. Let us assume that the bias current density $\vec{j}_{\text {ext }}$ flows in the $\hat{x}$ direction across the weak link of width $W$. Phenomenologically, Lorentz force drags flux lines moving with velocity $\vec{v}_{L}$ and viscosity $\eta$ along the $\hat{y}$ direction. For a viscous inertial flow in a homogeneous film of thickness $d$, the magnitudes of these vectors are related by:

$$
j_{\mathrm{ext}} \frac{\Phi_{0}}{c}=\eta v_{L}
$$

$\Phi_{0}=\mathrm{hc} / 2 e$ is the flux quantum. The flux flow resistivity $\rho_{f}=$ $R W / L$ is related to the viscosity by $[43,44]$ :

$$
\rho_{f}=\frac{E}{j_{\mathrm{ext}}}=H \frac{\Phi_{0}}{\eta c^{2}},
$$

where the compensation of the drift and the Lorentz force, $\vec{E}=\vec{H} \times \vec{v}_{L} / c$, for an inertial vortex flow, have been used in the second equality. 
In conventional type-II superconducting films, the magnetoresistance $R(H)$ is linear with $H$, which tells that $\eta$ is constant with $H$. This is not the case here for $\left|H_{a}\right|<H_{f}$, as shown in Fig. 5(b). In this range of fields, a parabola provides a rather good fit of $R(H)$.

Beyond $H_{f}$, there is a change of behavior and $R(H)$ becomes constant with the field. This implies that there is a regime of high viscosity and small resistance, $\eta \propto 1 / H$, for $\left|H_{a}\right|<H_{f}$ where $R(H)$ is roughly parabolic, and a regime of constant resistance with $\eta \propto H$ for larger applied fields. As, by increasing $H$, we expect that the density of unbound vortices increases, the crossover in Fig. 5(b) can be most likely attributed to a change in the dominant interaction between vortices.

To support this assumption, we have performed a classical simulation of 2D disks interacting via a long range Gaussian repulsive force and a short range quasihard core force. The details on the classical simulation can be found in Appendix. Such 2D classical simulations have been used in the context of the BKT phase transition [45] and the melting of a quasi-3Dvortex-glass with increasing $H_{a}$ at fixed temperature has been observed in YBCO [44,46], though at higher magnetic fields. This is not a proper melting, because a vortex-glass phase is not expected to take place at finite temperatures in a 2D layered structure as is the one discussed here.

Our simulation shows that a rather rigid gossamerlike texture forms at low $H$, when the density of vortices is rather low and the long range repulsion is dominant. We neglect disorder in our simulation, because an extended rigid texture with long range correlations cannot be pinned by the random configuration of pinning centers expected to be present under the Al pads. This would not be the case at higher temperatures because thermal fluctuations would soften the texture. It follows that the vortex structure can drift freely when the applied current acts as a force on it providing the dissipation mechanism. Numerically, we find $\rho_{f} \propto H^{2}$ at low $H$. By contrast, when $H$ increases, the density of free vortices increases, the long range correlation looses its dominant role, and the texture starts melting. In this regime, our simulation shows a saturation of the resistivity. Therefore, our simulation reproduces the observed magnetoresistance thus confirming our interpretation. Needless to say, all these features are a unique property of the geometry of the device and a straightforward consequence of the incipient BKT transition in the graphene sheet and cannot be found in thicker weak link films.

Having interpreted the quick collapse of $I_{c}$ when $\left|H_{a}\right|$ is turned on, we now focus on the revival of the Josephson current which is observed in Fig. 4 when $\left|H_{a}\right|$ is decreased. As mentioned already, our system can be regarded as granular, like a coated conductor [47,48]. In the simplest view, the grains correspond to the graphene area overlapping with the $\mathrm{Al}$ pads, and the grain boundary, across which the dissipative/nondissipative Josephson supercurrent flows, corresponds to the bare graphene weak link. In the critical state $[28,49]$ which forms in the "grains," vortices nucleate at the edges of the grains when $\left|H_{a}\right|$ increases and they attempt to move inside, toward the grain interior. Since pinning forces are opposed to vortex diffusion, a magnetic field gradient is formed in the grain with its resulting current profile. The recovering of the Josephson current when the sweeping of the magnetic
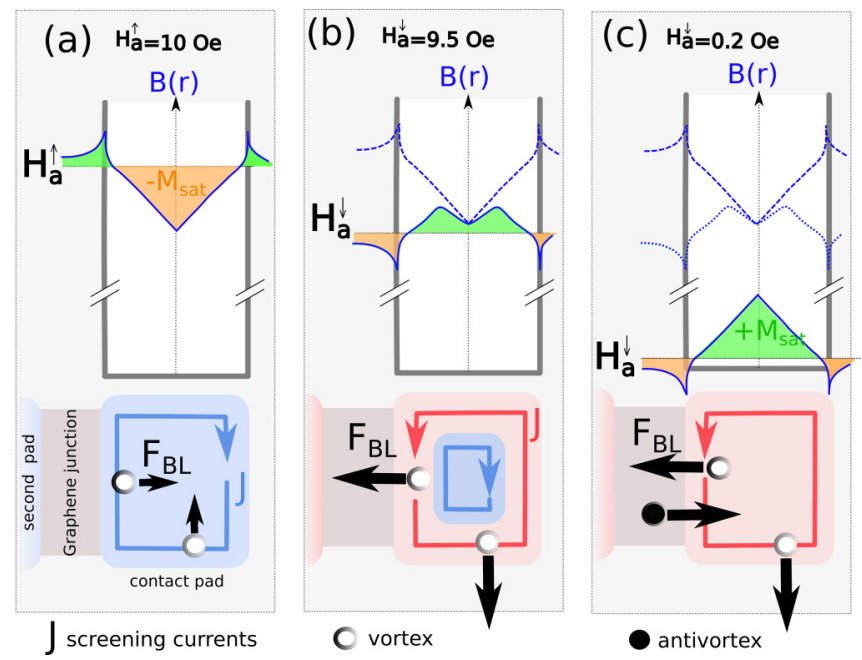

FIG. 6. Sketch of the vortex dynamics in the hysteresis of the Josephson current $I_{c}$ according to the CSM. The contacts are considered as grains. Just one of the contacts of the junction is drawn. Top: applied magnetic field $H_{a}$ and field induction $B(r)$ in the interior of the contact ( $B=0$ is at the bottom of the well). Bottom: Flowing screening currents densities $J_{c}$ (dark blue and dark red) and vortex (white circle) or antivortex (black circle) motion under the action of the Bean Livingston force, $F_{B L}$, which is due to the energy gradient named "Bean-Livingston barrier." (a) $H_{a} \simeq H_{m}$ : vortices enter the Al pads in increasing $\left|H_{a}\right|$. (b) $H_{a}<H_{m}$ : in decreasing $H_{a}$, the current density $J_{c}$ is reversed at the boundary and the Bean Livingston barrier is washed out, so that vortices exit from the pads. Since $H_{a}^{\downarrow}=9.5 \mathrm{Oe}>H_{m}-2 H^{*}$, an inverted cusp at the center of the magnetic field profile can be observed $\left(H^{*}\right.$ defines the field at which the contacts are fully penetrated, i.e., the central cusp still touches $B=0$ in (a)). (c) $H_{a} \simeq 0$ : vortices continue leaving the contact, while a negative return magnetic field builds up at its edges, which implies that some antivortices enter the contact and may annihilate with some of the exiting vortices.

field is inverted can be interpreted by considering the vortex dynamics in the grain region, see Fig. 6. Let us consider the $H_{a}>0$ case for the sake of the discussion. The same can be argued for the $H_{a}<0$ sweep. In increasing $H_{a}$, vortices continuously enter the grains overcoming the Bean-Livingston barrier [50,51]. This barrier is the sum of the contribution of the screening current (whose sign can be positive or negative depending if $\left|H_{a}\right|$ increases or decreases) and the image force (which does not depend on the direction of the magnetic field sweep). Vortices entering the grains produce penetration of the magnetic flux inside them. Due to the unequal diffusion inside the pad area, the field acquires a slope inside, which is approximately uniform according to the Bean CSM [see Fig. 6(a)]. At the very first moment when the magnetic field starts being reduced, those vortices which are loosely pinned to the defects are expelled immediately from the grains and swept away along the graphene "grain boundary," crossing the path of the Josephson current [Fig. 6(b)]. This is just the starting moment for the recovery of $I_{c}$. Further reduction of $H_{a}$ generates an inversion of the magnetic field gradient which penetrates the grains. This is accompanied by the inversion of the flow direction of the critical screening current at the grain boundaries, which, in turn, lowers the Bean-Livingston barrier $[50,51]$ for vortex flow out of the grains. 
The Bean-Livingston barrier is a known source of anomalies in the magnetization curves of type-II superconductors [52], because the barrier profile in the vicinity of the grain boundary differs in increasing or decreasing $\left|H_{a}\right|$.

Beyond this point, several tentative explanations can be proposed:

(i) only vortices which are depinned move along the grain boundary and are expelled. Vortices which remain pinned inside the grains do not contribute to the resistance. Figure 6(c) sketches what happens when sweeping $H_{a}$ down to zero field.

(ii) crowding of the vortices ejected out of the grains in the weak link channel with core repulsion between them can strongly increase viscosity in the channel and reduce their flowing across, so that the related flux flow resistance is also drastically reduced.

(iii) annihilation of some of the exiting vortices by antivortices of the broken $v-\bar{v}$ pairs created by thermal fluctuations or generated by the inversion of the local magnetic field at the grain boundary. All of this gives rise to the recovery of $I_{c}$ in decreasing $H_{a}$.

Let us comment now in more details why we observe that the maximum of $I_{c}$ is shifted to positive $H_{a} \approx 0.25$ Oe, when sweeping from positive fields down to zero field. According to the CSM, decreasing $H_{a}$ leaves a negative residual magnetic field $H_{r}^{A l}$ at the edges of the grains, which arises from the trapped magnetic field that survives and from the demagnetizing factors in the grain [see the magnetic field profile in Fig. 6(c)]. The local magnetic field in the weak link $H_{\text {loc }}^{\text {graphene }}=0$, at which the system presents the maximum of $I_{c}$, corresponds to the field at which $H_{a}=-H_{r}^{A l}$, because $H_{\text {loc }}^{\text {graphene }}=H_{a}+H_{r}^{A l}$. We performed magnetic fields sweeps with various $H_{m}$ in the range $0.5-3$ Oe and found that $H_{r}^{A l}$ saturates at $0.25 \mathrm{Oe}$ when $H_{m} \geqslant 1 \mathrm{Oe}$. If the cycle is continued and the magnetic field is reduced beyond $H_{\text {loc }}^{\text {graphene }}=0$, we find the collapse of $I_{c}$ once more and we enter the region of negative $H_{a}$ values (blue curve with increasing $\left|H_{a}\right|$ in Fig. 4).

Within the CSM, we can relate the value of $H_{r}^{A l}=$ $-0.25 \mathrm{Oe}$ to the field at which the Al pads are fully penetrated by the magnetic field, conventionally denoted by $H^{*}$. This is the magnetic field at which we can assume that most of the pinning centers in the $\mathrm{Al}$ pads have captured a flux line.

Following Refs. [47,48], we estimate

$$
H^{*}=H_{r}^{A l} \frac{n a}{x t},
$$

where $x$ and $n$ are numerical dimensionless demagnetization factors and $t$ and $a$ correspond to the thickness and to the linear size of each of the $\mathrm{Al}$ pads, respectively. In a first approximation, we have considered $a=4 \mu \mathrm{m}$ and we take $t$ as the thickness of the Al pad $(t=80 \mathrm{~nm})$. This provides a value of $H^{*}=0.5$ Oe just beyond the field $H_{f} \simeq 0.45$ Oe at which the collapse of $I_{c}$ is completed and the critical state is fully established. Therefore, for fields higher than $H^{*}$, a tiny Josephson current coexists with a sizable dissipation induced by the flow of free vortices.

Experimentally, the hysteresis can be observed up to magnetic fields as high as 20 Oe [see Fig. 4(a)]. At higher magnetic field, the $I_{c}(H)$ is reversible. This suggests that 20 Oe corresponds to the irreversibility field, i.e., the field at which the hysteresis and the critical state picture disappears, since the vortex lattice fully liquefies.

\section{FRAUNHOFER PATTERN OF THE JOSEPHSON JUNCTION}

In Figs. 7(a) and 7(b) we map the differential resistance $d V / d I$ of the J300-3 sample as a function of $H_{a}$ and $I$, for the two directions of the magnetic field sweep. The dark blue areas correspond to differential resistances $d V / d I$ below $5 \Omega$. The full curves in panels (a)-(d) are guides to the eye corresponding
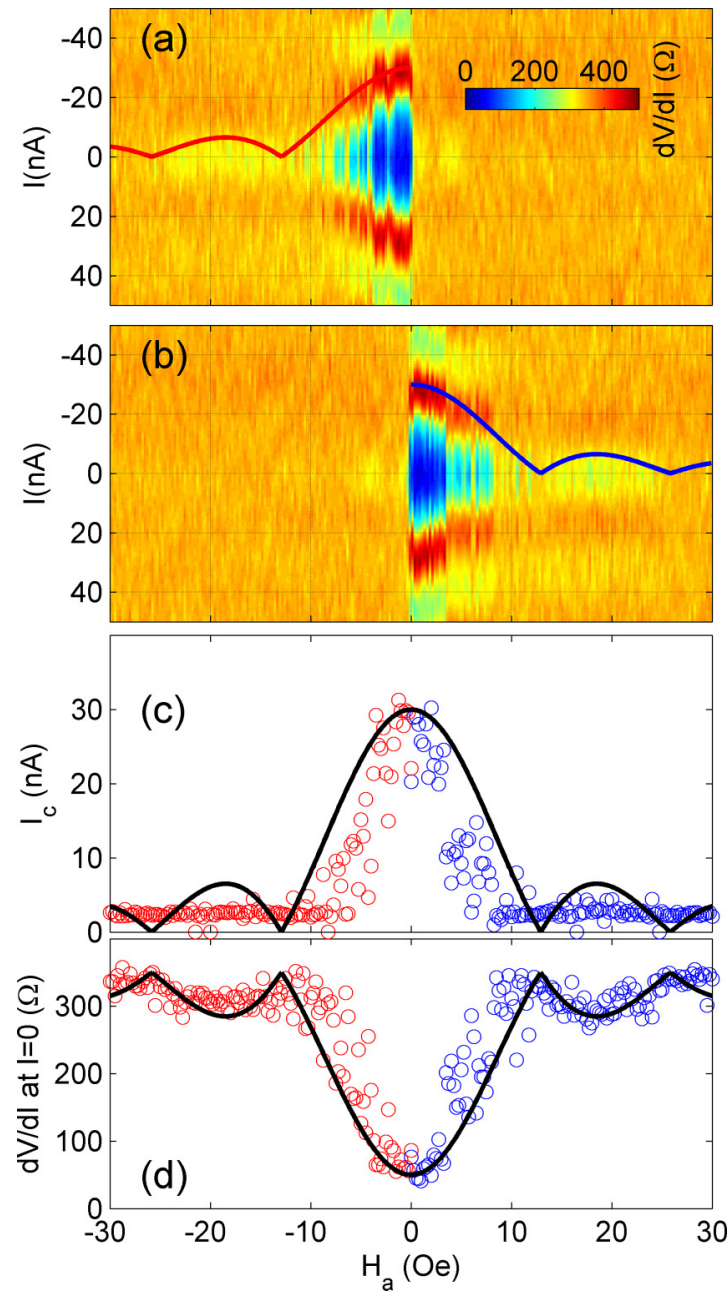

FIG. 7. The Fraunhofer pattern in junction J300-3. (a), (b) Color maps of the differential resistance $d V / d I(H, I)$ for the junction J300-3, evidencing a large hysteresis. (a) The magnetic field is swept from -30 to 30 Oe. (b) The field is swept from 30 to -30 Oe. The blue area corresponds to the low resistive region. The superposed blue and red curves are a Fraunhofer interference pattern, given as a reference, corresponding to a total area $S_{\text {eff }}=1.6 \mu \mathrm{m}^{2}$. (c) Critical current as a function of the magnetic field (red open circles: sweep from -30 to 0 Oe; blue open circles: sweep from 30 to 0 Oe). The black curve is the theoretical Fraunhofer pattern fit calculated using the RSJ model as explained in the main text. (d) The residual resistance at $I=0 \mathrm{nA}$ (red open circles: sweep from -30 to 0 Oe; blue open circles: sweep from 30 to $0 \mathrm{Oe}$ ) also reproduces the same Fraunhofer pattern, indicated by a thick black line as a guide for the eye. 

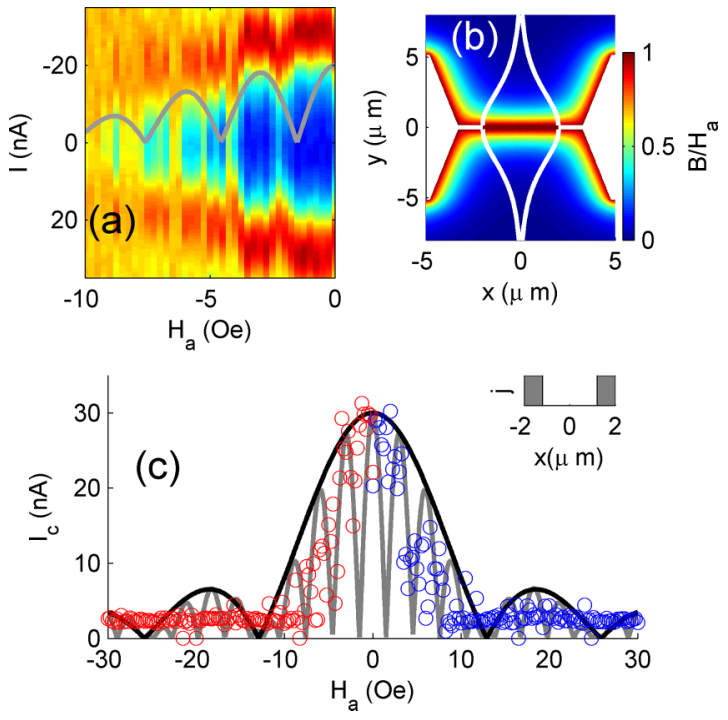

FIG. 8. The Rosenthal model. (a) Zoom of the color map of the differential resistance $d V / d I(H, I)$ for the junction of width $L \approx 300 \mathrm{~nm}$ presented in Fig. 7 , with the same color code. The data have been collected by decreasing the absolute value of the applied magnetic field. The dark blue area corresponds to the superconductive region. The superposed gray curve is a Fraunhofer interference pattern given as a reference, corresponding to an effective area $S_{\text {eff }}^{R} \simeq 9 \mu \mathrm{m}^{2}$. (b) Color map of the field penetration within the contacts, for a Pearl penetration length $\lambda=1 \mu \mathrm{m}$. The white line is a path enclosing the graphene junction which is perpendicular to the current flow. (c) Magnetic pattern. The fast oscillating $I_{c}$ in gray curve, as derived from the model in (b), with an inhomogeneous current density distribution $J_{c}$ (sketched in the inset), is added to the Fraunhofer pattern fit reported in Fig. 7(c). The current density distribution $J_{c}$ adopted in the fit is concentrated at the edges of the junction within two $200 \mathrm{~nm}$ wide strips. The same oscillations appear in (a) on a reduced scale.

to the Fraunhofer pattern usually appearing in extended junctions with uniform distribution of the supercurrent density $J_{c}$, giving $I_{c} \propto\left|\sin \left(\pi B S_{\mathrm{eff}} / \Phi_{0}\right) /\left(\pi B S_{\mathrm{eff}} / \Phi_{0}\right)\right|\left(\Phi_{0}=h c / 2 e\right.$ is the flux quantum) for an effective area $S_{\text {eff }} \simeq 1.6 \mu \mathrm{m}^{2}$. In panels (a) and (b), this Fraunhofer pattern fits roughly the experimental data obtained in decreasing $\left|H_{a}\right|$.

$I_{c}$, as obtained through the RSJ fit of the I-V curves (see Sec. II), is reported in Fig. 7(c) as a function of the applied magnetic field. By this method, the Fraunhofer pattern is less visible, while it is better retrieved in Fig. 7(d), which shows $d V / d I\left(H_{a}\right)$ at $I=0$ from the $\mathrm{I}-\mathrm{V}$ characteristics. The little shift of $H_{\text {loc }}^{\text {graphene }}=0$ discussed in the previous section cannot be appreciated on the magnetic field scale adopted here.

In Fig. 8 we zoom in the down sweep map of Fig. 7(a) and concentrate on the differential resistance. It appears clearly that the Fraunhofer oscillations considered up to now are just the envelope of a much faster oscillation pattern. This pattern is not strictly periodic with a pseudoperiod of 13 Oe [see Fig. 8(a)]. Occasionally, disturbances as jumps of the measured residual resistance at $I=0$ can also be spotted, probably due to flux jumps. This confirms that some vortex dynamics is taking place. We are unable to keep track of these microscopic irregular features, but we have set up a macroscopic point of view to account for the non-negligible magnetic field penetration in the $\mathrm{Al} / \mathrm{Ti}$ contacts. Indeed, the pseudoperiod of 1-3 Oe corresponds to a much larger effective area $S_{\text {eff }}^{R} \simeq 9 \mu \mathrm{m}^{2}$ than the weak link itself. We have calculated numerically the magnetic field profile for contacts in the thin film limit, following Rosenthal et al. [30], by solving the London equation $\nabla^{2} J_{s}-\lambda^{-2} J_{s}=0$ in a quasi-2D contact. A color map of the penetration of the field in the contacts appears in Fig. 8(b) with a penetration length $\lambda=1 \mu \mathrm{m}$ and a width of the weak link $W=4 \mu \mathrm{m}$. This value of the penetration length is significantly larger than the value usually reported for bulk Al (around a few tens of nanometers) and can be interpreted as a Pearl penetration length. The phase difference between two points is then calculated in the London gauge, by integrating the vector potential (which is proportional to the current) over a path which links these two points. We choose a path along which the longitudinal component of $J_{s}$ vanishes. This is plotted as the white curve in Fig. 8(b). The resulting enclosed area is $S_{\text {eff }}^{R} \sim W^{2} / 2 \simeq 8 \mu \mathrm{m}^{2}$, which is very close to the value extracted from the pseudoperiod. The fast oscillating gray curve in Fig. 8(c) is obtained by assuming an inhomogeneity in the critical current density $J_{c}$. Some inhomogeneity is expected, e.g., in planar devices where current focusing is typically observed in overdamped junctions at the edges of the junction [36,53]. The current density distribution adopted in the fit is drawn in the inset of Fig. 8(c). Both the fast $I_{c}$ oscillations [full gray curve in Fig. 8(c)] and the envelope modulation are retrieved by choosing $J_{c}$ concentrated in a strip $200 \mathrm{~nm}$ wide, at each of the boundaries of the graphene sheet. This model, which combines the London equation with an inhomogeneous current flow in a macroscopic approach, though remarkably sound, cannot reproduce some of the most puzzling characteristics of the conduction: the aperiodicity of the fast oscillations, the presence of flux jumps, not to speak about the hysteresis.

\section{DISCUSSION}

We have deposited a graphene monolayer on $\mathrm{SiC}$ by CVD and patterned various in-plane Josephson weak links on the same sample, with $\mathrm{Al} / \mathrm{Ti}$ thin contacts at a variable distance $L \gtrsim 200 \mathrm{~nm}$, as shown in Fig. 1. Details of the fabrication can be found in Ref. [19]. Each of the junctions can be thought of as an extended weak link, $W=4 \mu \mathrm{m}$ wide, between two thin metal grains. The junctions with $L \simeq 200-300 \mathrm{~nm}$ show Josephson conduction. Our analysis of $R(T)$ allows us to define a mean field critical temperature $T_{c 0}$ for our planar devices in the Aslamazov-Larkin paraconductivity regime precursive of superconductivity. The Halperin and Nelson interpolation formula for an incipient BKT transition captures the full temperature dependence of $R$ down to the operation temperature $(280 \mathrm{mK})$. The value of the dimensionless $b$ parameter which we get from the fit is quite large, of the order of the one found in the original formulation of the transition in the $2 \mathrm{D}-\mathrm{XY}$ model. Our $b$ value is also close to the one given in Ref. [40] for indium/indium oxide granular films. The ratio between the vortex core energy and the superfluid stiffness $J_{s}=\left(\phi_{0}^{2} / 4 \pi\right)\left(d / \lambda^{2}\right)$ that we find is $\mu / J_{s}=\pi^{2} \sqrt{b} / 4 \sim 5(d$ is the layer thickness and $\lambda$ is the London penetration length). Such a large ratio is related to the difference between $T_{c 0}$ and $T_{\mathrm{BKT}}$. Their separation is close to one order of magnitude, 
which is seldom found in $\mathrm{NbN}$ or $\mathrm{Al}$ films [54]. The superfluid stiffness is quite small because the inverse of the Pearl length appears for the graphene sheet, $\lambda_{P}=\lambda^{2} / d \sim 300 \mu \mathrm{m}$.

A Berezinskii-Kosterlitz-Thouless transition at lower temperature, $T \sim 100 \mathrm{mK}$, allows us to interpret the apparently odd dependence of the Josephson current on magnetic field. This can be attributed to the dynamics of the free interacting vortices hosted by the graphene sheet which is quite extended under the Al/Ti pads. Vortices originated by broken $v-\bar{v}$ vortex pairs penetrate the overlapping aluminum pads and can be pinned by the impurities, including unavoidable defects due to photoresist residue.

Figures 4 and 7 entail the unique features of these structures, in which the Josephson critical current $I_{c}$ is hysteretic, when cycling with an applied magnetic field $H_{a}$, after a ZFC. The Josephson current, though sensitive to the phase difference modulated by the magnetic field, is always accompanied by some dissipation. The latter is strong when increasing $\left|H_{a}\right|$, but it is quite low when reverting the sweeping of the field. We attribute the hysteresis to the presence of a large number of impurity centers at the $\mathrm{Al}$ pads which pin the vortices that are pushed into the dirty metal. The S-N-S structure resembles a granular material with the graphene gap playing the role of a grain boundary [43]. The $\mathrm{Al} / \mathrm{Ti}$ pads enter a critical state that can be described by the Bean CSM. Decreasing $\left|H_{a}\right|$, loosely pinned vortices are expelled out of the $\mathrm{Al}$ grains. In addition, reversal of the current at the boundary of the Al pads produces a lowering of the Bean-Livingston barrier for vortex expulsion, thus enhancing the process. This vortex dynamics implies a reduction in the flux flow across the graphene weak link and a recovery of the Josephson critical current $I_{c}$. The hysteresis is fully reproducible.

The Fraunhofer-like pattern for the Josephson critical current can be fitted within the Rosenthal model. The physical phenomena at the origin of the supposed inhomogeneous current flow invoked to justify the fast oscillating pattern with magnetic field may have many different origins. For instance, current focused at the edges of the junction is typically observed in overdamped Josephson dynamics [36]. We conclude that, in the granular picture of Sec. V there are two different length scales: a microscopic scale with vortex pinning and a macroscopic scale defined by $\lambda \simeq 1 \mu \mathrm{m}$, which defines the macroscopic magnetization of the Al pads and the hysteretic behavior of the supercurrent. The fit of the Fraunhofer-like pattern that we obtain with the Rosenthal model is rather satisfactory. Still, field penetration in the CSM and London equation in the Rosenthal model for the weak link are not enough to explain all the features of the experiment. Additional features like the nonperiodicity of the fast oscillations in the Fraunhofer pattern or some occasional flux jumps appearing in the data cannot be captured by the continuum macroscopic picture.

A remarkable feature of the magnetic sweep, when starting from zero field in the $H_{a} \lesssim 0.45$ Oe narrow range, is the drastic collapse of $I_{c}$. This is a unique property of the geometry of the device (we have checked that point contacts in which $W$ is much shorter do not show the collapse) and we attribute it to the incipient BKT transition in the graphene sheet. We figure out that in this range of $H$ values, the free vortices are quite dilute at $280 \mathrm{mK}$ but, as they originate from breaking of $v-\bar{v}$ vortex pairs due to thermal fluctuations, they are long range correlated. A gossamerlike texture is created in the whole extension of the graphene sheet. Such a solid array is rigid because elastic vibrations of the flux lines are expected to be frozen at $280 \mathrm{mK}$. At the beginning they cannot move freely, as long as the applied magnetic field is so weak that there is no unbalance between the number of vortices and antivortices. However, with increasing $H_{a}$, the Lorentz force induced by the flowing current starts drifting rigidly the texture in which vortex of one charge prevail, in the orthogonal direction, generating a flux flow resistance. As long as the texture is rigid, it cannot get pinned by the impurities, because of incommensurability between the random space distribution of pinning centers and the vortex texture. Indeed, the pinning force vanishes in the average. The flux flow produces the collapse of $I_{c}$.

By increasing the density of free vortices, the effect of the long range correlations is reduced and the liquefaction of the texture starts. In Fig. 5, we are monitoring a "weak first order transition" induced by the quasi-2D contacts [46].

We have simulated this dynamics with the classical diffusion of disks interacting via a long range potential. It is important to stress that this transition occurs with increasing density of free vortices and has no connection with the melting transition that occurs with temperature in HTc anisotropic superconductors. The latter is due to thermal vibration of flux lines [44]. Vortices in the liquid can feel the pinning forces individually and give origin to the critical state and to the hysteresis. The energetics of vortex lines entering or exiting a bulk type II superconductor has been extensively considered in the past, in connection with the determination of $H_{c 1}[50,51,55]$ and goes under the name of "Bean-Livingston barrier" for vortex penetration in the bulk of a superconductor. We have calculated the Bean-Livingston barrier by solving the London equation in the planar structure in the framework of the Rosenthal model [30] by adding the vortex and its image, and we have found that the barrier is rather small in our planar structure because it is thin and screening is quite low. Moreover, the barrier disappears when the current is reversed at the boundary and this has the consequence that the recovery of $I_{c}$, when the field sweeping is inverted, is quite fast.

\section{SUMMARY}

To sum up, we have measured diffusive transport in a coplanar graphene Josephson junction with a single layer (or, locally, very few) graphene sheet extending under the $\mathrm{Al}$ contacts on an insulating $\mathrm{SiC}$ background. The Josephson current, the differential resistance and the response to the magnetic field has been measured down to $300 \mathrm{mK}$. We find:

(a) $R(T)$ curves which can be fitted with the celebrated formula derived by Halperin and Nelson for a temperature range in the crossover from paraconductivity below $1 \mathrm{~K}$, down to Berezinskii-Kosterlitz-Thouless superconductivity with a critical temperature $T_{\mathrm{BKT}}$ of the order of $0.1 \mathrm{~K}$ (see Fig. 3).

(b) The parameters deduced from the fit in the BKT picture suggest that the condensation energy lost in the vortex core is more than five times larger than the phase superconducting stiffness (which is estimated from the screening length $\lambda>1 \mu \mathrm{m})$. 
(c) Phase coherent Josephson conduction is measured, provided the separation between the Al banks is $<400 \mathrm{~nm}$, although in the presence of a small resistance.

(d) An unexpected fully reproducible magnetic hysteresis is found: The Josephson current $I_{c}(H)$ collapses with $H$ upramping as soon as the field is turned on, and undergoes revival as soon as the ramping of $H$ is inverted (see Fig. 4). For higher fields ( $H>20 \mathrm{Oe}$ ), the sweeping is reversible.

(e) The plots $I_{c}(H)$ are symmetrical and $I_{c}$ oscillates in the revival, in a way that recalls the Fraunhofer interference pattern.

(f) An interpretation of the hysteresis and of the flux flow resistance is given within the critical state model, which rests on vortex dynamics with their penetration into the Al banks.

Finally, this work demonstrates that CVD on $\mathrm{SiC}$ can provide decisive progress towards scalability of superconducting graphene junctions with immediate applicative impact. Unusual properties of the planar measured structures originate from the superconductive proximity involving graphene. This study paves the way to the design of devices having exactly the same barrier quality together with constructive parameters that can be selectively changed, in search for the desired functionalities. This is the only possible path for a hybrid graphene/superconductor technology.

\section{ACKNOWLEDGMENTS}

Enlightening discussions with L. Benfatto, V. Bouchiat, P. Brouwer, J.-R. Huntzinger, and Y. V. Kopelevich are gratefully acknowledged. Work supported by PICS CNRSCNR 2014-2016 Transport phenomena and Proximity-induced Superconductivity in Graphene junctions, Swedish Foundation for Strategic Research (SSF) under the project "Graphene based high frequency electronics," FIRB HybridNanoDev RBFR1236VV (Italy) and by EU FP7, under grant agreement no 604391 Graphene Flagship.

\section{APPENDIX: CLASSICAL SIMULATION OF VORTEX DYNAMICS}

We simulate a random walk of vortices with the thermal Brownian dynamics of $N=2048$ disks interacting in 2D space via the potential

$$
u(r)=\epsilon\left[\left(\frac{\sigma}{r}\right)^{2}+e^{-r^{2} / \ell^{2}}\right] .
$$

Here $\epsilon$ is the unit of energy, and $\ell=8 \sigma(\sigma$ is the length unit, practically corresponding to the disk diameter). The disks

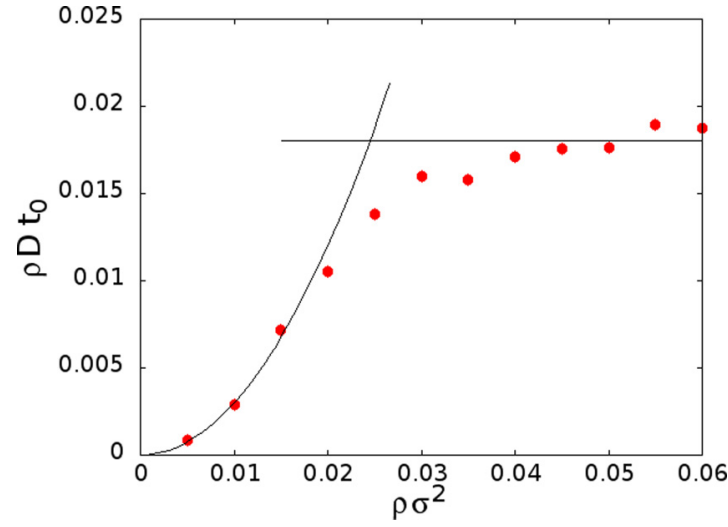

FIG. 9. Plot of $\rho D t_{0}$ as a function of $\rho \sigma^{2}$ for a classical Brownian motion of interacting disks with interaction potential given by Eq. (A1). By trading the model for an analogy with the dynamics of the vortex liquid at low temperature, this is a plot of the resistivity versus magnetic field (i.e., $e^{2} \rho_{f} / \hbar d$ vs $H \xi^{2} / \phi_{0}$, where $d$ is the thickness of the weak link layer).

move in a $2 \mathrm{D}$ square box of side $L=\sqrt{N / \rho}$, where $\rho$ is the density of the disks. Periodic boundary conditions have been used. The unit of time is $t_{0}=\sigma \sqrt{M / \epsilon}$ where $M$ is the mass of the disks. The free particle diffusivity is $D_{0}=\sigma^{2} / t_{0}$. After thermalization, at a temperature $T=0.012 \epsilon$, we extract the diffusion coefficient $D$ of the interacting disks. Due to the Einstein relation, the diffusivity $D \propto k_{B} T / \eta d$ is inversely proportional to the viscosity $\eta$. Here $d$ is the thickness of the weak link layer. In Fig. 9 we plot the product $\rho D t_{0}$ as a function of $\rho \sigma^{2}$. We find a low density regime in which the long range Gaussian interaction is dominant and the diffusion coefficient increases with the density. At higher densities, on the other hand, the short range repulsion becomes dominant, and the diffusion coefficient decreases roughly linearly with the density, giving rise to a constant product $\rho D t_{0}$.

To mimic the vortex dynamics, we assume that $\rho \sigma^{2}$ of the disks is proportional to $H \xi^{2} / \phi_{0}$. Here the coherence length $\xi$ plays the role of the radius of the vortex core. The corresponding horizontal scale in the plot of Fig. 9 shows that the crossover occurs at $H_{a} \sim 0.5$ Oe if the vortex core $\xi \sim 1.3 \mu \mathrm{m}$. This points to quite extended Pearl vortices which are of no surprise in our structure. Next, we trade $\hbar / t_{0}$ for $k_{B} T$ in the Einstein relation so that:

$$
\rho D t_{0} \rightarrow \frac{\hbar}{\eta d} \frac{H}{\phi_{0}}=\frac{(2 e)^{2}}{\hbar} R,
$$

where $R=\rho_{f} / d$ and Eq. (4) has been used in the last equality to connect the viscosity $\eta$ to the flux flow resistivity $\rho_{f}$.
[1] Y.-J. Doh, J. A. van Dam, A. L. Roest, E. P. A. M. Bakkers, L. P. Kouwenhoven, and S. De Franceschi, Science 309, 272 (2005).

[2] D. Bercioux and P. Lucignano, Rep. Prog. Phys. 78, 106001 (2015).

[3] A. H. Castro Neto, F. Guinea, N. M. R. Peres, K. S. Novoselov, and A. K. Geim, Rev. Mod. Phys. 81, 109 (2009).
[4] C. W. J. Beenakker, Rev. Mod. Phys. 80, 1337 (2008).

[5] X.-L. Qi and S.-C. Zhang, Rev. Mod. Phys. 83, 1057 (2011).

[6] M. Z. Hasan and C. L. Kane, Rev. Mod. Phys. 82, 3045 (2010).

[7] F. S. Bergeret, A. F. Volkov, and K. B. Efetov, Rev. Mod. Phys. 77, 1321 (2005).

[8] A. I. Buzdin, Rev. Mod. Phys. 77, 935 (2005). 
[9] M. Veldhorst, M. Snelder, M. Hoek, T. Gang, V. K. Guduru, X. L. Wang, U. Zeitler, W. G. van der Wiel, A. A. Golubov, H. Hilgenkamp, and A. Brinkman, Nat. Mater. 11, 417 (2012).

[10] C. Kurter, A. D. K. Finck, P. Ghaemi, Y. S. Hor, and D. J. Van Harlingen, Phys. Rev. B 90, 014501 (2014).

[11] L. Galletti, S. Charpentier, M. Iavarone, P. Lucignano, D. Massarotti, R. Arpaia, Y. Suzuki, K. Kadowaki, T. Bauch, A. Tagliacozzo, F. Tafuri, and F. Lombardi, Phys. Rev. B 89, 134512 (2014).

[12] E. M. Stoudenmire, J. Alicea, O. A. Starykh, and M. P. A. Fisher, Phys. Rev. B 84, 014503 (2011).

[13] I. Affleck and D. Giuliano, J. Stat. Phys. 157, 666 (2014).

[14] J. Alicea, Rep. Prog. Phys. 75, 076501 (2012).

[15] C. Beenakker, Ann. Rev. Condens. Matter Phys. 4, 113 (2013).

[16] G. Campagnano, P. Lucignano, D. Giuliano, and A. Tagliacozzo, J. Phys.: Condens. Matter 27, 205301 (2015).

[17] V. L. Berezinskii, Sov. Phys. JETP 34, 610 (1972).

[18] J. M. Kosterlitz and D. J. Thouless, J. Phys. C 6, 1181 (1973).

[19] B. Jouault, S. Charpentier, D. Massarotti, A. Michon, M. Paillet, J.-R. Huntzinger, A. Tiberj, A. Zahab, T. Bauch, P. Lucignano, A. Tagliacozzo, F. Lombardi, and F. Tafuri, J. Supercond. Nov. Magn. 29, 1145 (2016).

[20] J. Kedzierski, P.-L. Hsu, P. Healey, P. Wyatt, C. Keast, M. Sprinkle, C. Berger, and W. A. de Heer, IEEE Trans. Electron Devices 55, 2078 (2008).

[21] C. W. J. Beenakker, Phys. Rev. Lett. 97, 067007 (2006).

[22] M. Titov, A. Ossipov, and C. W. J. Beenakker, Phys. Rev. B 75, 045417 (2007).

[23] A. M. Black-Schaffer and S. Doniach, Phys. Rev. B 78, 024504 (2008).

[24] T. Dirks, T. L. Hughes, S. Lal, B. Uchoa, Y. F. Chen, C. Chialvo, P. M. Goldbart, and N. Mason, Nat. Phys. 7, 386 (2011).

[25] M. Ben Shalom, M. J. Zhu, V. I. Fal'ko, A. Mishchenko, A. V. Kretinin, K. S. Novoselov, C. R. Woods, K. Watanabe, T. Taniguchi, A. K. Geim, and J. R. Prance, Nat. Phys. 12, 318 (2016).

[26] N. Mizuno, B. Nielsen, and X. Du, Nat. Commun. 4, 2716 (2013).

[27] V. E. Calado, S. Goswami, G. Nanda, M. Diez, A. R. Akhmerov, K. Watanabe, T. Taniguchi, T. M. Klapwijk, and L. M. K. Vandersypen, Nat. Nanotechnol. 10, 761 (2015).

[28] C. P. Bean, Phys. Rev. Lett. 8, 250 (1962).

[29] B. M. Kessler, C. O. Girit, A. Zettl, and V. Bouchiat, Phys. Rev. Lett. 104, 047001 (2010).

[30] P. A. Rosenthal, M. R. Beasley, K. Char, M. S. Colclough, and G. Zaharchuk, Appl. Phys. Lett. 59, 3482 (1991).

[31] A. Michon, S. Vézian, A. Ouerghi, M. Zielinski, T. Chassagne, and M. Portail, Appl. Phys. Lett. 97, 171909 (2010).

[32] B. Jabakhanji, A. Michon, C. Consejo, W. Desrat, M. Portail, A. Tiberj, M. Paillet, A. Zahab, F. Cheynis, F. Lafont, F. Schopfer, W. Poirier, F. Bertran, P. Le Fèvre, A. Taleb-Ibrahimi, D. Kazazis, W. Escoffier, B. C. Camargo, Y. Kopelevich, J. Camassel, and B. Jouault, Phys. Rev. B 89, 085422 (2014).
[33] G. Giovannetti, P. A. Khomyakov, G. Brocks, V. M. Karpan, J. van den Brink, and P. J. Kelly, Phys. Rev. Lett. 101, 026803 (2008).

[34] L. Longobardi, D. Massarotti, G. Rotoli, D. Stornaiuolo, G. Papari, A. Kawakami, G. P. Pepe, A. Barone, and F. Tafuri, Phys. Rev. B 84, 184504 (2011).

[35] D. Stornaiuolo, G. Rotoli, D. Massarotti, F. Carillo, L. Longobardi, F. Beltram, and F. Tafuri, Phys. Rev. B 87, 134517 (2013).

[36] A. Barone and G. Paternò, Physics and Applications of the Josephson Effect (John Wiley \& Sons, New York, 1982).

[37] J. M. Martinis and R. L. Kautz, Phys. Rev. Lett. 63, 1507 (1989).

[38] J. M. Kivioja, T. E. Nieminem, J. Claudon, O. Buisson, F. W. J. Hekking, and J. P. Pekola, Phys. Rev. Lett. 94, 247002 (2005); J. Männik, S. Li, W. Qiu, W. Chen, V. Patel, S. Han, and J. E. Lukens, Phys. Rev. B 71, 220509 (2005); D. Massarotti, L. Longobardi, L. Galletti, D. Stornaiuolo, D. Montemurro, G. Pepe, G. Rotoli, A. Barone, and F. Tafuri, Low Temp. Phys. 38, 263 (2012).

[39] L. G. Aslamasov and A. I. Larkin, Phys. Lett. A 26, 238 (1968).

[40] A. T. Fiory, A. F. Hebard, and W. I. Glaberson, Phys. Rev. B 28, 5075 (1983).

[41] B. Halperin and D. R. Nelson, J. Low Temp. Phys. 36, 599 (1979).

[42] I. V. Borzenets, U. C. Coskun, S. J. Jones, and G. Finkelstein, Phys. Rev. Lett. 107, 137005 (2011).

[43] L. Ji, M. S. Rzchowski, N. Anand, and M. Tinkham, Phys. Rev. B 47, 470 (1993).

[44] M. Tinkham, Introduction to Superconductivity (McGraw-Hill, Singapore, 1996).

[45] S. Prestipino, F. Saija, and P. V. Giaquinta, Phys. Rev. Lett. 106, 235701 (2011).

[46] R. H. Koch, V. Foglietti, W. J. Gallagher, G. Koren, A. Gupta, and M. P. A. Fisher, Phys. Rev. Lett. 63, 1511 (1989).

[47] A. Palau, T. Puig, X. Obradors, E. Pardo, C. Navau, A. Sanchez, A. Usoskin, H. C. Freyhardt, L. Fernndez, B. Holzapfel, and R. Feenstra, Appl. Phys. Lett. 84, 230 (2004).

[48] A. Palau, T. Puig, X. Obradors, and C. Jooss, Phys. Rev. B 75, 054517 (2007).

[49] E. Zeldov, J. R. Clem, M. McElfresh, and M. Darwin, Phys. Rev. B 49, 9802 (1994).

[50] C. P. Bean and J. D. Livingston, Phys. Rev. Lett. 12, 14 (1964).

[51] P. G. De Gennes, Superconductivity of Metals and Alloys, Advanced book classics (Perseus, Cambridge, MA, 1999).

[52] V. N. Kopylov, A. E. Koshelev, I. F. Schegolev, and T. G. Togonidze, Physica C 170, 291 (1990).

[53] S. Hart, H. Ren, T. Wagner, P. Leubner, M. Muhlbauer, C. Brune, H. Buhmann, L. W. Molenkamp, and A. Yacoby, Nat. Phys. 10, 638 (2014)

[54] J. Yong, T. R. Lemberger, L. Benfatto, K. Ilin, and M. Siegel, Phys. Rev. B 87, 184505 (2013).

[55] A. Y. Aladyshkin, A. V. Silhanek, W. Gillijns, and V. V. Moshchalkov, Supercond. Sci. Technol. 22, 053001 (2009). 